\title{
Gravitation as Geometry or as Field
}

\author{
Walter Petry \\ Mathematical Institute of the University Duesseldorf, Duesseldorf, Germany \\ Email: wpetry@meduse.de
}

How to cite this paper: Petry, W. (2017) Gravitation as Geometry or as Field. Journal of Applied Mathematics and Physics, $\mathbf{5}$, 862-872.

https://doi.org/10.4236/jamp.2017.54076

Received: January 22, 2017

Accepted: April 27, 2017

Published: April 30, 2017

Copyright $\odot 2017$ by author and Scientific Research Publishing Inc. This work is licensed under the Creative Commons Attribution International License (CC BY 4.0).

http://creativecommons.org/licenses/by/4.0/ c) (i) Open Access

\begin{abstract}
General relativity (GR) and gravitation in flat space-time (GFST) are covariant theories to describe gravitation. The metric of GR is given by the form of proper-time and the metric of GFST is a flat space-time form different from that of proper-time. The source of GR is the matter tensor and the Einstein tensor describes the gravitational field. The source of GFST is the total energymomentum including gravitation. The field is described by a non-linear differential operator of order two in divergence form. The results of the two theories agree for weak gravitational fields to the order of measurable accuracy. It is well-known that homogeneous, isotropic, cosmological models of GR start from a point singularity of the universe, the so called big bang. The density of matter is infinite. Therefore, our observable big universe implies an expansion of space, in particular an inflationary expansion in the beginning. Doubts are stated because infinities don't exist in physics. An explanation to the present, controversial discussion of expanding accelerating or non-accelerating universe as well as non-expanding universe is given. GFST starts in the beginning from a homogeneous, isotropic universe with uniformly distributed energy and no matter. In the course of time matter is created out of energy where the total energy is conserved. There is no singularity, i.e. no big bang. The space is flat and non-expanding.
\end{abstract}

\section{Keywords}

Gravitation, Cosmology, Flat Space, No Singularity, No Big Bang, Non-Expanding Universe

\section{Introduction}

Einstein's general theory of relativity is at present the most accepted theory of gravitation. The theory gives for weak gravitational fields, agreement with the corresponding experimental results. But the results for homogeneous, isotropic, cosmological models imply difficulties. So, the universe starts from a point singularity, i.e. the universe starts from a point with infinite density of matter. The 
observed universe is very big. Hence, the space of the universe must expand very quickly which implies the introduction of an inflationary universe in the beginning. There are controversial discussions about the universe, e.g. is the universe accelerating or not. GFST uses a pseudo-Euclidean geometry and the proper time is defined similar to that of general relativity, i.e. space-time and proper time are different from one another. GFST starts from an invariant Lagrangian which gives by standard methods, the field equations of gravitation. The source is the total energy-momentum tensor including gravitation. The energy-momentum of gravitation is a tensor. The field is described by non-linear differential equations of order two in divergence form. The theory is generally covariant. The gravitational equations together with the conservation law of the total energy-momentum give the equations of motion for matter. The application of the theory implies for weak gravitational fields the same results as GR to experimental accuracy, e.g. gravitational red shift, deflection of light, perihelion precession, radar time delay, post-Newtonian approximation, gravitational radiation of a two-body system and the precession of the spin axis of a gyroscope in the orbit of a rotation body. But there are also differences of the results of these two theories. GFST gives non-singular, cosmological models. The covariance of GFST and the existence of non-singular cosmological models imply the possibility to interpret the solutions as expanding or as non-expanding space yielding an accelerating resp. non-expanding universe. GFST may e.g. be found in the book [1] and in the cited references. Additionally, non-singular, cosmological models are e.g. given in the articles [2] [3] [4] [5] [6].

Subsequently, homogeneous, isotropic, cosmological models will be summarized. Let us use the pseudo-Euclidean geometry. The resulting universe is nonsingular under the assumption that the sum of the density parameters is greater than one, e.g. a little bit greater than one. It starts without matter and without radiation and all the energy is gravitational energy. Matter and radiation emerge from this energy by virtue of the conservation of the total energy. The space is flat and the interpretation of a non-expanding space is natural. But it is also possible to state an expansion of space by a suitable transformation as consequence of general covariance of the equations. Matter and radiation are generated from the beginning of the universe and the universe becomes hot. A certain time after the beginning matter and radiation decrease and the universe converges to dark energy as time goes to infinity. Hence, a universe given by GFST appears more natural than that received by GR which gives singular solution with infinite densities. The geometry of GR is in general non-Euclidean but the observed universe implies a flat space.

GR is well-known in contrast to GFST. Therefore, GFST and resulting cosmological models are shortly summarized in the next two sections. All these results can be found in the article [5].

Section 2 contains GFST; Section 3 contains cosmological models; Section 4 contains GR and Section 5 states GFST. Cosmological models of GR and GFST are compared with one another. 


\section{GFST}

The theory of GFST is shortly summarized. The metric is the flat space-time given by

$$
(\mathrm{ds})^{2}=-\eta_{i j} \mathrm{~d} x^{i}
$$

where $\left(\eta_{i j}\right)$ is a symmetric tensor. Pseudo-Euclidean geometry has the form

$$
\left(\eta_{i j}\right)=(1,1,1,-1) \text {. }
$$

Here, $\left(x^{i}\right)=\left(x^{1}, x^{2}, x^{3}\right)$ are the Cartesian coordinates and $x^{4}=c t$. Let

$$
\eta=\operatorname{det}\left(\eta_{i j}\right) \text {. }
$$

The gravitational field is described by a symmetric tensor $\left(g_{i j}\right)$. Let $\left(g^{i j}\right)$ be defined by

$$
g_{i k} g^{k j}=\delta_{i}^{j}
$$

and put similarly to (3)

$$
G=\operatorname{det}\left(g_{i j}\right) .
$$

The proper time $\tau$ is defined by

$$
(c \mathrm{~d} \tau)^{2}=-g_{i j} \mathrm{~d} x^{i} \mathrm{~d} x^{j} .
$$

The Lagrangian of the gravitational field is given by

$$
L(G)=-\left(\frac{-G}{-\eta}\right)^{1 / 2} g_{i j} g_{k l} g^{m n}\left(g_{/ m}^{i k} g_{/ n}^{j l}-\frac{1}{2} g_{/ m}^{i j} g_{/ n}^{k l}\right)
$$

where the bar/denotes the covariant derivative relative to the flat space-time metric (1).

The Lagrangian of dark energy (given by the cosmological constant $\Lambda$ ) has the form

$$
L(\Lambda)=-8 \Lambda\left(\frac{-G}{-\eta}\right)^{1 / 2}
$$

Let

$$
\kappa=4 \pi k / c^{4}
$$

and of matter of a perfect fluid are where $\kappa$ is the gravitational constant. Then, the mixed energy-momentum tensor of gravitation, of dark energy and of matter of a perfect fluid are

$$
\begin{gathered}
T(G)_{j}^{i}=\frac{1}{8 \kappa}\left[\left(\frac{-G}{-\eta}\right)^{1 / 2} g_{k l} g_{m n} g^{i r}\left(g_{/ j}^{k m} g_{/ r}^{l n}-\frac{1}{2} g_{/ j}^{k l} g_{/ r}^{m n}\right)+\frac{1}{2} \delta_{j}^{i} L(G)\right] \\
T(\Lambda)_{j}^{i}=\frac{1}{16 \kappa} \delta_{j}^{i} L(\Lambda) \\
T(M)_{j}^{i}=(\rho+p) g_{j k} u^{k} u^{i}+\delta_{j}^{i} p c^{2} .
\end{gathered}
$$

Here, $\rho, p$ and $u^{i}$ denote density, pressure and four-velocity of matter. It holds by (6) 


$$
c^{2}=-g_{i j} u^{i} u^{j}
$$

Define the covariant differential operator

$$
D_{j}^{i}=\left[\left(\frac{-G}{-\eta}\right)^{1 / 2} g^{k l} g_{j m} g_{/ l}^{m i}\right]_{/ k}
$$

of order two. Then, the field equations for the potentials $\left(g_{i j}\right)$ have the form

$$
D_{j}^{i}-\frac{1}{2} \delta_{j}^{i} D_{k}^{k}=4 \kappa T_{j}^{i}
$$

where the total energy-momentum is the sum of the energy-momentum tensors of matter, gravitation and cosmological constant, i.e.,

$$
T_{j}^{i}=T(G)_{j}^{i}+T(M)_{j}^{i}+T(\Lambda)_{j}^{i} .
$$

Define the symmetric energy-momentum tensor

$$
T(M)^{i j}=g^{i k} T(M)_{k}^{j} .
$$

Then, the equations of motion in covariant form are

$$
T(M)_{i / k}^{k}=\frac{1}{2} g_{k l / i} T(M)^{k l} .
$$

In addition to the field Equations (13) and the equations of motion (16) the conservation law of the total energy-momentum holds, i.e.

$$
T_{i / k}^{k}=0 \text {. }
$$

The field equations of gravitation are formally similar to those of GR where $T_{j}^{i}$ is the energy-momentum without that of gravitation since the energy-momentum of gravitation is not a tensor for GR. Furthermore, the differential operator is the Einstein tensor which may give a non-Euclidean geometry.

The results of this chapter may be found in the book [1] and in many other articles of the author, as e.g. in [5].

\section{Homogeneous, Isotropic, Cosmological Models}

In this chapter GFST is applied to homogeneous, isotropic, cosmological models. The pseudo-Euclidean geometry (1) with (2) is used. The matter tensor is given by perfect fluid with velocity

$$
u^{i}=0(i=1,2,3)
$$

and pressure $p$ and density $\rho$ with

$$
\begin{aligned}
& p=p_{m}+p_{r}, \\
& \rho=\rho_{m}+\rho_{r}
\end{aligned}
$$

where the indices $m$ and $r$ denote matter and radiation. The equations of state for matter (dust) and radiation are

$$
p_{m}=0, p_{r}=\frac{1}{3} \rho_{r} .
$$

The potential are by virtue of (18) and the homogeneity and isotropy

$$
g_{i j}=\left\{\begin{array}{lc}
a^{2}(t) & (i=j=1,2,3) \\
-1 / h(t) & (i=j=4) \\
0 & (i \neq j) .
\end{array}\right.
$$


The four-velocity is by Equation (18) and Equation (6)

$$
\left(u^{i}\right)=\left(0,0,0, c h^{1 / 2}\right) \text {. }
$$

Let $t_{0}=0$ be the present time and assume as initial conditions at present

$$
a(0)=h(0)=1, \dot{a}(0)=H_{0}, \dot{h}(0)=\dot{h}_{0}, \rho_{m}(0)=\rho_{m 0}, \rho_{r}(0)=\rho_{r 0}
$$

where the dot denotes the time derivative, $H_{0}$ is the Hubble constant and $\dot{h}_{0}$ is a further constant, $\rho_{m 0}$ and $\rho_{r 0}$ denote the present densities of matter and radiation. It follows from (16) under the assumption that matter and radiation do not interact

$$
\rho_{m}=\rho_{m 0} / h^{1 / 2}, \rho_{r}=3 p_{r}=\rho_{r 0} /\left(a h^{1 / 2}\right) .
$$

The field Equation (13) implies by the use of (21) the two nonlinear differential equations

$$
\begin{gathered}
\frac{\mathrm{d}}{\mathrm{d} t}\left(a^{3} h^{1 / 2} \frac{\dot{a}}{a}\right)=2 \kappa c^{4}\left(\frac{1}{2} \rho_{m}+\frac{1}{3} \rho_{r}+\frac{\Lambda}{2 \kappa c^{2}} \frac{a^{3}}{h^{1 / 2}}\right), \\
\frac{\mathrm{d}}{\mathrm{d} t}\left(a^{3} h^{1 / 2} \frac{\dot{h}}{h}\right)=4 \kappa c^{4}\left(\frac{1}{2} \rho_{m}+\rho_{r}+\frac{1}{8 \kappa c^{2}} L(G)-\frac{\Lambda}{2 \kappa c^{2}} \frac{a^{3}}{h^{1 / 2}}\right)
\end{gathered}
$$

where

$$
L(G)=\frac{1}{c^{2}} a^{3} h^{1 / 2}\left(-6\left(\frac{\dot{a}}{a}\right)^{2}+6 \frac{\dot{a}}{a} \frac{\dot{h}}{h}+\frac{1}{2}\left(\frac{\dot{h}}{h}\right)^{2}\right) .
$$

The expression $\frac{1}{16 \kappa} L(G)$ is the density of gravitation. The conservation law of the total energy gives

$$
\left(\rho_{m}+\rho_{r}\right) c^{2}+\frac{1}{16 \kappa} L(G)+\frac{\Lambda}{2 \kappa} \frac{a^{3}}{h^{1 / 2}}=\lambda c^{2}
$$

where $\lambda$ is a constant of integration. Equations (25), (26) and (27) give by the use of the $i$ initial conditions (23)

$$
\frac{\dot{h}}{h}=-6 \frac{\dot{a}}{a}+2 \frac{4 \kappa c^{4} \lambda t+\varphi_{0}}{2 \kappa c^{4} \lambda t^{2}+\varphi_{0} t+1}
$$

with

$$
\varphi_{0}=3 H_{0}\left(1+\frac{1}{6} \frac{\dot{h_{0}}}{H_{0}}\right)
$$

Integration of (28) yields

$$
a^{3} h^{\frac{1}{2}}=2 \kappa c^{4} \lambda t^{2}+\varphi_{0} t+1
$$

Equation (27) gives for the present time $t_{0}=0$ by the use of the initial conditions (23)

$$
\frac{1}{3}\left(8 \kappa c^{4} \lambda-\varphi_{0}^{2}\right)=4\left[\frac{8}{3} \pi k\left(\rho_{m 0}+\rho_{r 0}+\frac{\Lambda c^{2}}{8 \pi \mathrm{k}}\right)-H_{0}^{2}\right] .
$$


It follows from (27) by the use of the standard definition of the density parameters of matter, radiation and the cosmological constant with the abbreviation

$$
K_{0}=\left(\Omega_{m}+\Omega_{r}+\Omega_{\Lambda}\right) / \Omega_{m}
$$

the differential equation

$$
\left(\frac{\dot{a}}{a}\right)^{2}=\frac{H_{0}^{2}}{\left(2 \kappa c^{4} \lambda t^{2}+\varphi_{0} t+1\right)^{2}}\left[-\Omega_{m} K_{0}+\Omega_{r} a^{2}+\Omega_{m} a^{3}+\Omega_{\Lambda} a^{6}\right] .
$$

The initial condition is by (23)

$$
a(0)=1 \text {. }
$$

The solution of (33) with (30) describes a homogeneous, isotropic, cosmological model by GFST.

Relation (31) can be rewritten in the form

$$
\frac{8 \kappa c^{4} \lambda}{H_{0}^{2}}-\left(\frac{\varphi_{0}}{H_{0}}\right)^{2}=12 \Omega_{m} K_{0} .
$$

A necessary and sufficient condition to avoid singular solutions of (33) is

$$
K_{0}>0
$$

which yields

$$
2 \kappa c^{4} \lambda t^{2}+\varphi_{0} t+1>0
$$

for all $t \in \mathbb{R}$. Hence, condition (35) implies a non-singular solution for all $t \in \mathbb{R}$, i.e. we get a non-singular cosmological model. It exists a $t_{1}<t_{0}=0$ such that

$$
\dot{a}\left(t_{1}\right)=0
$$

Put $a_{1}=a\left(t_{1}\right)$ then it follows from (33a) with $t=t_{1}$

$$
\Omega_{r} a_{1}^{2}+\Omega_{m} a_{1}^{3}+\Omega_{m} a_{1}^{6}=\Omega_{m} K_{0} .
$$

It holds for all $t \in \mathbb{R}$

$$
a(t) \geq a_{1}>0 .
$$

Subsequently assume

$$
a_{1} \ll a(0)=1 .
$$

Then we get by virtue of (38)

$$
K_{0} \ll 1
$$

It follows from (32) by virtue of (41)

$$
\Omega_{r}+\Omega_{m}+\Omega_{\Lambda}=1+\Omega_{m} K_{0},
$$

i.e. the sum of the density parameters is a little bit greater than one. Hence, $a(t)$ starts from a positive value, decreases to a small positive value, and then increases for all $t \in \mathbb{R}$.

The proper time from the beginning of the universe till time $t$ is

$$
\tilde{\tau}(t)=\int_{-\infty}^{t} 1 / h^{1 / 2}(t) \mathrm{d} t .
$$


The differential Equation (33a) is rewritten by the use of (30) in the form

$$
\left(\frac{\dot{a}}{a}\right)^{2}=H_{0}^{2} \frac{1}{h}\left(-\frac{\Omega_{m} K_{0}}{a^{6}}+\frac{\Omega_{r}}{a^{4}}+\frac{\Omega_{m}}{a^{3}}+\Omega_{\Lambda}\right) .
$$

Hence, the differential equation for the function $a$ by the use of the proper time is

$$
\left(\frac{1}{a} \frac{\mathrm{d} a}{\mathrm{~d} \tilde{\tau}}\right)^{2}=H_{0}^{2}\left(-\frac{\Omega_{m} K_{0}}{a^{6}}+\frac{\Omega_{r}}{a^{4}}+\frac{\Omega_{m}}{a^{3}}+\Omega_{\Lambda}\right) .
$$

This differential equation is by virtue of (41) and a not too small function $a(t)$ identical with that of GR for a flat homogeneous, isotropic universe. Therefore, away from the beginning of the universe, the result for the universe agrees for GFST with that of GR. Under the above stated assumptions and $\Omega_{r}=0$ the differential Equation (33) can analytically be solved. It follows that $a(t)$ starts from a small positive value at $-\infty$ and then it decreases for increasing $t$ to $a_{1}>0$ at $t_{1}$. Finally it increases for $t>t_{1}$ to infinity as $t$ goes to infinity. Relation (30) gives positive values $h(t)$ for all $t . h(t)$ starts from infinity at $-\infty$, decreases to a positive value and then it increases to infinity as $t$ goes to infinity. The longer calculations are omitted and they can be found in the article [3].

The differential Equations (44) and (45) show that the condition (35) is important to avoid singularities. GR gives $K_{0}=0$ which yields the singularity of the model (big bang). We introduce in addition to the proper time $\tilde{\tau}$ the absolute time $t^{\prime}$ by

$$
\mathrm{d} t^{\prime}=\frac{1}{a(t) h^{1 / 2}(t)} \mathrm{d} t=\frac{1}{a(t)} \mathrm{d} \tilde{\tau}
$$

This gives for the proper time in the universe

$$
(c \mathrm{~d} \tau)^{2}=-a(t)^{2}\left[|\mathrm{~d} x|^{2}-\left(\mathrm{d} c t^{\prime}\right)^{2}\right]
$$

where $|\mathrm{d} x|$ denotes the Euclidean norm of the vector $\mathrm{d} x=\left(\mathrm{d} x_{1}, \mathrm{~d} x_{2}, \mathrm{~d} x_{3}\right)$.

Relation (47) implies that the absolute value of the light-velocity is equal to vacuum light-velocity $c$ for all times $t^{\prime}$.

The introduction of the absolute time $t^{\prime}$ in the differential Equation (45) gives

$$
\left(\frac{\mathrm{d} a}{\mathrm{~d} t^{\prime}}\right)^{2}=\frac{H_{0}^{2}}{a^{2}}\left(-\Omega_{m} K_{0}+\Omega_{r} a^{2}+\Omega_{m} a^{3}+\Omega_{\Lambda} a^{6}\right) .
$$

Assume that a light ray is emitted at distance $r$ at time $t_{e}^{\prime}$ resp. at time $t_{e}^{\prime}+\mathrm{d} t_{e}^{\prime}$ and it is received by the observer at time $t^{\prime}$ resp. at time $t^{\prime}+\mathrm{d} t^{\prime}$. Then, it follows

$$
\begin{aligned}
& r=\int_{t_{e}^{\prime}}^{t^{\prime}} c \mathrm{~d} t^{\prime}=c\left(t^{\prime}-t_{e}^{\prime}\right), \\
& r=\int_{t_{e}^{\prime}+\mathrm{d} t_{e}^{\prime}}^{t^{\prime}+\mathrm{d} t^{\prime}} c \mathrm{~d} t^{\prime}=c\left(t^{\prime}+\mathrm{d} t^{\prime}-t_{e}^{\prime}-\mathrm{d} t_{e}^{\prime}\right) .
\end{aligned}
$$

These two equations imply 


$$
\mathrm{d} t^{\prime}=\mathrm{d} t_{e}^{\prime} .
$$

The age of the universe since the minimal value of $a(t)$ measured with absolute time $t^{\prime}$ till now

$$
\begin{aligned}
\Delta t^{\prime} & =\int_{t_{1}^{\prime}}^{t_{0}^{\prime}} \mathrm{d} t^{\prime}=\int_{a_{1}}^{1} 1 /\left(\frac{\mathrm{d} a}{\mathrm{~d} t^{\prime}}\right) \mathrm{d} a=\frac{1}{H_{0}} \int_{a_{1}}^{1} a \mathrm{~d} a /\left(-\Omega_{m} K_{0}+\Omega_{r} a^{2}+\Omega_{m} a^{3}+\Omega_{\Lambda} a^{6}\right)^{1 / 2} \\
& \geq \frac{1}{H_{0}} \int_{a_{1}}^{1} a \mathrm{~d} a /\left(-\Omega_{m} K_{0}+\left(\Omega_{r}+\Omega_{m}+\Omega_{\Lambda}\right) a^{2}\right)^{1 / 2} \approx \frac{1}{H_{0}} .
\end{aligned}
$$

Therefore, the age of the universe measured with absolute time is greater than $1 / H_{0}$ independent of the density parameters, i.e. there is no age problem.

We will now calculate the red shift of light emitted from a distant object at rest and received by the observer at present time. It is useful to introduce the absolute time. Assume that an atom at a distant object emits a photon at time $t_{e}^{\prime}$. It follows from relation (46)

$$
\mathrm{d} \tilde{\tau}=a\left(t_{e}^{\prime}\right) \mathrm{d} t^{\prime}
$$

Therefore, the energy of the emitted photon is

$$
E \sim-g_{44} \frac{\mathrm{d} t^{\prime}}{\mathrm{d} \tau} \sim a\left(t_{e}^{\prime}\right) E_{0} .
$$

The energy of the photon moving to the observer in the universe is constant by virtue of (47), i.e. by the constant light velocity. Then, the corresponding received frequency is

$$
v=a\left(t_{e}^{\prime}\right) v_{0}
$$

where $v_{0}$ is the frequency emitted at the observer from the same atom. The red shift is given by

$$
z=v_{0} / v-1=1 / a\left(t_{e}^{\prime}\right)-1
$$

Light emitted at distance $r$ at time $t_{e}^{\prime}$ and received at $r=0$ at time $t_{0}^{\prime}$ has by the constant velocity of light the relation

$$
r=c\left(t_{0}^{\prime}-t_{e}^{\prime}\right)
$$

This gives by Taylor expansion of $a\left(t_{e}^{\prime}\right)$ in relation (51)

$$
z=H_{0} \frac{r}{c}+\left(1-\frac{1}{2} \frac{1}{H_{0}^{2}} \frac{\mathrm{d}^{2} a\left(t_{0}^{\prime}\right)}{\mathrm{d} t_{0}^{\prime 2}}\right)\left(H_{0} \frac{r}{c}\right)^{2} .
$$

Differentiation of equation (48) yields by neglecting small expressions

$$
\frac{\mathrm{d}^{2} a\left(t_{e}^{\prime}\right)}{\mathrm{d} t_{e}^{\prime 2}} \approx H_{0}^{2}\left(1-\frac{1}{2} \Omega_{m}+\Omega_{\Lambda}\right) .
$$

This gives the red shift formula

$$
z=H_{0} \frac{r}{c}+\frac{3}{4} \Omega_{m}\left(H_{0} \frac{r}{c}\right)^{2} .
$$

The detailed calculations of Formula (52) can be found in the book [1].

Higher order Taylor expansion gives higher order red shift approximations. The red shift is already derived in the article [11] without Doppler Effect but 
only by gravitation.

\section{General Relativity}

The theory of general relativity as well as the resulting cosmological models is well-known. Astronomical observations show that the universe is flat. Therefore, only flat space of general theory is stated. The curvature of the universe must be zero by the cosmological principle. This means that the sum of the density parameters is equal to one. The strong gravitational field in the neighbourhood of the singularity implies a high curvature which contradicts to a flat universe, i.e. with curvature zero. This problem is solved partly by the introduction of an inflationary expansion. Hence, either general relativity is not correct or the cosmological principle is not valid.

\section{Field theory of Gravitation}

GFST is a field theory which describes gravitation as a field in flat space-time. The theory is covariant and it is studied in the book [1], in the cited references there in and in the articles [2] [3] and [4]. This theory gives for weak gravitational fields to the lowest order of accuracy (measurable accuracy) the same results as general relativity. But there are differences to general relativity for strong gravitational fields, e.g. for the universe in the beginning. The source of the field equations of gravitation is the total energy-momentum including that of gravitational field which is a tensor for this theory. The universe starts without matter in the beginning and consists only of (gravitational) energy. In the course of time matter and radiation are created where the total energy is conserved. Singularities don't exist under the assumption that the sum of the density parameters is greater than one (at least a little bit greater which is subsequently assumed). Hence, there is no big bang. Models with and without cosmological constant are studied in the book [1]. By the use of the pseudo-Euclidean geometryas metric the solution yields a non-expanding universe. The red shift of distant objects in a non-expanding universe was already given in article [11]. It is worth to mention that by virtue of the covariance of the theory the non-singular results can be interpreted in a non-expanding and in an expanding space. The space of the theory is flat independent of the density parameters. The presently assumed density parameter of matter is $\approx 0.3$. To avoid singular solutions of the cosmological model the density parameter of the cosmological constant must be $\approx 0.7$ such that the sum of the two values is a little bit greater than one.

The present discussion of the universe about non-expanding or expanding with acceleration can be solved by GFST because non-expanding space seems to be the natural interpretation but the interpretation as expanding space is also possible. GR demands by virtue of the point -singularity an acceleration of the universe.

Article [10] contains further differences of the two theories.

A theory of gravitation in flat space-time (GFST) is given. The field is a tensor of rank 2 which is described on a flat space-time metric, e.g. the pseudo-Euclid- 
ean geometry. The field equations have as source the total energy-momentum tensor inclusive that of gravitation which is a tensor. The conservation of the total energy-momentum tensor implies the equations of motion and reverse. The theory is generally covariant and the results of GFST and general relativity (GR) agree for weak fields to the lowest order of approximation. Homogeneous, isotropic, cosmological models of GFST are studied in the pseudo-Euclidean geometry. Assuming that the sum of the density parameters is a little bit greater than one the resulting cosmological models are non-singular. In the beginning of the universe no matter exists, i.e. all the energy is gravitation. In the course of time matter and radiation are generated from gravitational energy. The total energy is conserved. The space is flat and non-expanding. Certain time after the beginning the results of the two theories highly agree with one another under the assumption that the universe is flat. The general covariance of the theory gives the possibility to interpret the results in a non-expanding or in an expanding universe.

\section{Conclusions}

GFST is a field theory like Electrodynamics and GR is geometry. For weak fields, the two theories give approximately the same results under the assumption that the universe is flat. Astrophysical observations show that the universe is flat. Cosmological models of GR imply a singularity in the beginning of the universe with infinite matter density (big bang). Hence, in the neighbourhood of the singularity, there is a high curvature, i.e. space is not flat in the neighbourhood of the singularity. The cosmological principle implies that space is everywhere flat. Hence, we get a contradiction to GR or to the cosmological principle. The universe starts from a point-singularity. Therefore, space must expand or even inflationary expand by virtue of the big observed universe.

GFST is generally covariant, i.e. the space can be interpreted as non-expanding or as expanding. The density parameter of matter is at present assumed to be $\approx 0.3$. Therefore, the density parameter of dark energy is $\approx 0.7$ with the assumption that the sum of the density parameter is a little bit greater than one to imply non-singular cosmological models. Cosmological models of GR have a flat space under the assumption that the sum of the density parameters is equal to one. Therefore, GR and GFST give about the same values for the density parameters. But in the beginning of the universe, the solutions of GR and GFST are quite different. There exists a singularity (big bang) by GR and the solution of GFST is everywhere defined and regular, i.e. no bang. It is worth to mention that singularities are physically not allowed.

\section{References}

[1] Petry, W. (2014) A Theory of Gravitation in Flat Space-Time. Science PG 2014.

[2] Petry, W. (1981) Cosmological Models without Singularities. General Relativity and Gravitation, 13, 1057-1071. https://doi.org/10.1007/BF00756365

[3] Petry, W. (1990) Nonsingular Cosmological Model with Matter Creation and En- 
tropy Production. General Relativity and Gravitation, 22, 1045-1965. https://doi.org/10.1007/BF00757815

[4] Petry, W. (1997) On the Hubble Law in a Nonexpanding Nonstationary Universe with Cosmological Constant. Astrophysics and Space Science, 254, 305-317. https://doi.org/10.1023/A:1000938931517

[5] Petry, W. (2013) Cosmology with Bounce by Flat Space-Time Theory of Gravitation and a New Interpretation. Journal of Modern Physics, 4, 20-25. https://doi.org/10.4236/jmp.2013.47A1003

[6] Petry, W. (2014) Gravitation in Flat Space-Time and General Relativity. Journal of Applied Mathematics Physics, 2, 50-54.

[7] Lerner, E. (2005) Evidence for a Non-Expanding Universe: Surface Brightness Data from HUDF. AIP Conference Proceedings, 822, 60. arXiv: astro-ph/0509611 https://doi.org/10.1063/1.2189123

[8] Petry, W. (2013) Modified Hubble Law. Physics Essays, 26, 315-320. https://doi.org/10.4006/0836-1398-26.2.315

[9] Petry, W. (2015) Creation of a Non-Expanding, Non-Singular Universe. Journal of Modern Physics, 6, 1085-1094. https://doi.org/10.4236/jmp.2015.68113

[10] Petry, W. (2016) Comparing Gravitation in Flat Space-Time with General Relativity. Journal of Modern Physics, 7, 1492-1499.

https://doi.org/10.4236/jmp.2016.712135

[11] Petry, W. (2007) Is the Universe Really Expanding? arXiv: 0705.4359

\section{Submit or recommend next manuscript to SCIRP and we will provide best} service for you:

Accepting pre-submission inquiries through Email, Facebook, LinkedIn, Twitter, etc. A wide selection of journals (inclusive of 9 subjects, more than 200 journals)

Providing 24-hour high-quality service

User-friendly online submission system

Fair and swift peer-review system

Efficient typesetting and proofreading procedure

Display of the result of downloads and visits, as well as the number of cited articles

Maximum dissemination of your research work

Submit your manuscript at: http://papersubmission.scirp.org/

Or contact jamp@scirp.org 